\title{
Different Neural Substrates Mediate Cocaine Seeking after Abstinence versus Extinction Training: A Critical Role for the Dorsolateral Caudate-Putamen
}

\author{
Rita A. Fuchs, ${ }^{2}$ R. Kyle Branham, ${ }^{1}$ and Ronald E. See ${ }^{1}$ \\ ${ }^{1}$ Department of Neurosciences, Medical University of South Carolina, Charleston, South Carolina 29425, and 2Department of Psychology, University of \\ North Carolina, Chapel Hill, North Carolina 27599-3270
}

\begin{abstract}
Cue-induced reinstatement of extinguished drug seeking is a preclinical model of relapse. However, relapse typically occurs after abstinence rather than explicit extinction training. We show that inactivation of the dorsolateral caudate-putamen, but not other structures previously implicated in reinstatement, attenuates cocaine seeking after abstinence. This suggests that there is limited overlap in the substrates of cocaine seeking after abstinence versus extinction, and that habit learning exerts greater control over drug seeking than regions implicated in stimulus-reward associations.
\end{abstract}

Key words: dorsal striatum; caudate-putamen; cocaine; self-administration; reinstatement; extinction; abstinence

\section{Introduction}

Exposure to drug-associated stimuli elicits craving and increases the likelihood of relapse in cocaine users (O’Brien et al., 1998). The extinction/reinstatement paradigm is a preclinical model used to investigate the neural mechanisms of relapse (Shalev et al., 2002). In this model, animals respond for drug infusions in a distinct context or in the presence of discrete stimuli. The animals then undergo extinction training (no drug or cues) to decrease response rates and demonstrate reinstatement of drug seeking in response to drug-associated cues. However, relapse in humans typically occurs after a drug-free period, or abstinence, as opposed to explicit extinction training. Extinction not only reduces the baseline rate of responding but also suppresses the possible contribution of several exogenous variables (i.e., habit, stress, and exploratory behavior) toward lever pressing and produces neuroadaptive changes (Sutton et al., 2003). Thus, different neural substrates likely mediate cocaine seeking after extinction versus abstinence.

The functional integrity of several structures, including the basolateral amygdala (BLA) and dorsomedial prefrontal cortex (dmPFC), is necessary for explicit cue- or context-induced reinstatement of extinguished cocaine seeking (McLaughlin and See, 2003; Fuchs et al., 2005). The present study assessed the effects of inactivation of these structures on cocaine seeking after abstinence. The focus of investigation was also extended to the dorsolateral caudate-putamen $(\mathrm{dlCPu})$, a region that undergoes exten-

Received Dec. 2, 2005; revised Feb. 7, 2006; accepted Feb. 28, 2006.

This work was supported by National Institute on Drug Abuse Grants DA10462, DA15369, and DA17673 and National Institutes of Health Grant C06 RR015455. We thank Jacqueline McGinty and Peter Kalivas for their insightfu comments, as well as Jennifer Jordan-Bear, Macon Parker, Jordan Case, and Ritu Mehta for competent technical assistance.

Correspondence should be addressed to Ronald E. See, Medical University of South Carolina, Department of Neurosciences, 173 Ashley Avenue, Suite 416B, Charleston, SC 29425. E-mail: seere@musc.edu.

D01:10.1523/JNEUROSCI.5146-05.2006

Copyright $\odot 2006$ Society for Neuroscience $\quad$ 0270-6474/06/263584-05\$15.00/0 sive reorganization during habit learning (Jog et al., 1999) that may underlie compulsive drug seeking (Gerdeman et al., 2003).

\section{Materials and Methods}

Subjects. Male Sprague Dawley rats $(n=69$; Charles River Laboratories, Wilmington, MA), weighing 300-325 g at the start of the experiment, were individually housed in a temperature- and humidity-controlled vivarium on a reversed light/dark cycle. Rats were maintained on 20-25 $\mathrm{g}$ of rat chow per day and ad libitum water. The housing and treatment of the rats followed the guidelines of the Guide for the Care and Use of Laboratory Rats (Institute of Laboratory Animal Resources on Life Sciences, National Research Council, 1996). Rats were given $5 \mathrm{~d}$ for adaptation before the start of the experiment.

Food training. Rats were trained to lever press on a fixed ratio 1 (FR1) schedule of food reinforcement ( $45 \mathrm{mg}$ pellets; Noyes, Lancaster, $\mathrm{NH}$ ) in self-administration chambers $(30 \times 20 \times 24 \mathrm{~cm}$ high; Med Associates, St. Albans, VT) during a $16 \mathrm{~h}$ overnight training session. The chambers were equipped with two retractable levers, a stimulus light above each lever, a food pellet dispenser, a house light on the wall opposite to the levers, and a speaker connected to a tone generator (ANL-926; Med Associates). During the session, each active lever press resulted in delivery of a food pellet only. Lever presses on the inactive lever had no programmed consequences. After food training, pellet dispensers were removed from the chambers.

Surgery. Forty-eight hours after lever training, rats were anesthetized using ketamine and xylazine ( 66.6 and $1.3 \mathrm{mg} / \mathrm{kg}$, i.p., respectively) followed by equithesin ( $1 \mathrm{ml} / \mathrm{kg}$, i.p., of a solution of $9.72 \mathrm{mg} / \mathrm{ml}$ pentobarbital sodium, $42.5 \mathrm{mg} / \mathrm{ml}$ chloral hydrate, and $21.3 \mathrm{mg} / \mathrm{ml}$ magnesium sulfate heptahydrate dissolved in a $44 \%$ propylene glycol and $10 \%$ ethanol). Indwelling catheters were constructed as described previously (Fuchs et al., 2004b). The end of the catheter was inserted into the right jugular vein. The catheter ran subcutaneously and exited on the rat's back, posterior to the shoulder blades.

Immediately after the catheter surgery, the rats received bilateral stainless steel guide cannulas (26 gauge; Plastics One, Roanoke, VA) aimed dorsal to the BLA $[-2.7 \mathrm{~mm}$ anteroposterior (AP), $\pm 5.0 \mathrm{~mm}$ mediolateral (ML), $-6.6 \mathrm{~mm}$ dorsoventral (DV)], dmPFC ( $+3.0 \mathrm{~mm} \mathrm{AP,} \pm 0.6 \mathrm{~mm} \mathrm{ML}$, $-1.6 \mathrm{~mm} \mathrm{DV})$, and $\mathrm{dlCPu}(+1.2 \mathrm{~mm} \mathrm{AP}, \pm 3.8 \mathrm{~mm} \mathrm{ML},-3.4 \mathrm{~mm} \mathrm{DV})$ 
using standard stereotaxic procedures. Three small screws and cranioplastic cement secured the guide cannulas to the skull. Stylets (Plastics One) were placed into the cannulas and catheter to prevent occlusion.

To extend catheter patency, the catheters were flushed daily for $5 \mathrm{~d}$ after surgery with $0.1 \mathrm{ml}$ of cefazolin $(10.0 \mathrm{mg} / \mathrm{ml}$; Schein Pharmaceutical, Florham Park, NJ) dissolved in heparinized saline (70 U/ml; ElkinsSinn, Cherry Hill, NJ). Thereafter, catheters were flushed with $0.1 \mathrm{ml}$ of heparinized saline $(10 \mathrm{U} / \mathrm{ml})$ before each self-administration session and with $0.1 \mathrm{ml}$ of the cefazolin solution and $0.1 \mathrm{ml}$ of heparinized saline after each session. Catheter patency was periodically verified by infusing $0.08-$ $0.10 \mathrm{ml}$ of methohexital sodium $(20 \mathrm{mg} / \mathrm{ml}$, i.v.; Eli Lilly \& Co., Indianapolis, IN), which produces a rapid loss of muscle tone.

Cocaine self-administration. Self-administration was conducted during $2 \mathrm{~h}$ sessions on consecutive days during the rats' dark cycle and continued until the rats self-administered a minimum of 10 infusions per session for a minimum of $10 \mathrm{~d}$ (acquisition criterion). Rats were trained to lever press according to an FR1 schedule of cocaine reinforcement (cocaine hydrochloride; $0.2 \mathrm{mg} / 0.05 \mathrm{ml}$; National Institute on Drug Abuse, Research Triangle Park, NC). After each infusion, responses on the active lever had no consequences during a $20 \mathrm{~s}$ timeout period. Responses on the inactive lever had no programmed consequences. Data collection and reinforcer delivery were controlled using MedPC software version IV (Med Associates).

Intracranial drug infusions. For intracranial infusions, injection cannulas ( 33 gauge; Plastics One) were inserted to a depth of $1 \mathrm{~mm}$ (dmPFC) or $2 \mathrm{~mm}$ (dlCPu and BLA) below the tip of the guide cannulas. A combination of baclofen and muscimol (B/M) $(0.1$ and $1.0 \mathrm{~mm}$, respectively) or PBS vehicle were infused bilaterally into each region over $2 \mathrm{~min}$, using $10 \mu \mathrm{l}$ Hamilton (Reno, NV) syringes and an infusion pump (Harvard Apparatus, South Natick, MA). Dose-response analyses revealed that this concentration of $\mathrm{B} / \mathrm{M}$ elicits maximal suppression of cocaineprimed reinstatement (McFarland and Kalivas, 2001) and inhibits conditioned-cued reinstatement after infusion at volumes of $0.3-0.6 \mu \mathrm{l} /$ side (Fuchs et al., 2004a,b). As an alternative to multiple infusion sites, infusion volume was adjusted to result in larger areas of drug spread in larger brain regions. Infusions were made bilaterally at a volume of 0.3 ( $\mathrm{dmPFC}), 0.5$ (BLA), or $0.6(\mathrm{dlCPu}) \mu \mathrm{l} /$ hemisphere over $2 \mathrm{~min}$. The infusion cannulas were left in place for $1 \mathrm{~min}$ before and after the infusion.

Experiment 1: inactivation effects on reinstatement of cocaine-seeking behavior after abstinence. Rats $(n=29)$ were trained to self-administer cocaine, whereby active lever presses resulted in a $2 \mathrm{~s}$ activation of the infusion pump only. The house light was illuminated throughout each session. After the last self-administration day, rats remained in the colony room on post-cocaine days $1-7$. On post-cocaine days $8-14$, rats were transported to a procedure room that was distinctly different from the self-administration testing room and were placed into clear plastic holding chambers equipped with filter tops $(27 \times 16 \times 20 \mathrm{~cm}$ high; Allentown Caging, Allentown, PA) for $2 \mathrm{~h} / \mathrm{d}$. Daily handling and exposure to the alternate environment were conducted to minimize the impact of dishabituation on cocaine seeking on subsequent test days without extinguishing the motivational effects of the cocaine-paired environment. To accustom rats to the intracranial infusion procedure, injection cannulas were bilaterally inserted into the guide cannulas for $4 \mathrm{~min}$, but fluid was not infused, before placement into the alternate environment on post-cocaine day 11 .

On post-cocaine days 15 and 19, the rats were transported to the self-administration testing room. The rats received bilateral microinfusions of $\mathrm{B} / \mathrm{M}$ or vehicle into the $\mathrm{BLA}(n=10), \operatorname{dmPFC}(n=11)$, or $\mathrm{dlCPu}$ $(n=8)$ immediately before placement into the previously cocaine-paired (i.e., self-administration) context. Using a within-subjects design, the order of $\mathrm{B} / \mathrm{M}$ and vehicle infusions was counterbalanced across two test sessions based on cocaine intake history. During testing, lever presses were recorded but had no scheduled consequences. On the days intervening test sessions, the rats were exposed to the alternate environment. The length of the inter-test interval was determined based on previous reinstatement studies, in which rats needed an average of $3 \mathrm{~d}$ to reach the extinction criterion and become eligible for additional testing.

Experiment 2: inactivation effects on context-induced reinstatement of cocaine seeking after extinction. In experiment 1 , inactivation of the dl-
$\mathrm{CPu}$, but not the BLA or dmPFC, attenuated cocaine-seeking behavior in a drug-associated context after abstinence. These findings were interesting because we have shown that the BLA and dmPFC play critical roles in context-induced reinstatement of cocaine seeking after extinction (Fuchs et al., 2005). Experiment 2 extended this line of research by assessing the potential contribution of the dlCPu to context-induced reinstatement of cocaine seeking after extinction.

A separate group $(n=8)$ received guide cannula implants into the dlCPu. In addition, an additional group $(n=13)$ had bilateral cannulas implanted in the overlying somatosensory cortex (SS) (1.2 mm AP, \pm 5.0 $\mathrm{mm} \mathrm{ML},-2.2 \mathrm{~mm} \mathrm{DV}$ ), which served as an anatomical control region to the $\mathrm{dlCPu}$. Infusion parameters were the same as described above for the $\mathrm{dlCPu}$. Subjects were trained to self-administer cocaine in one of two distinctly different contexts. Context 1 consisted of a modified operant conditioning chamber that contained a red house light, a clicking auditory stimulus $(78 \mathrm{~dB}, 10 \mathrm{~Hz})$, and a vanilla-scented odor dish beneath a black, rough-textured floorboard. Context 2 consisted of a chamber that contained a blinking, white stimulus light, a pure tone auditory stimulus $(78 \mathrm{~dB}, 2 \mathrm{kHz})$, and a sandalwood-scented odor dish beneath a clear, smooth floorboard. These salient contextual stimuli were presented throughout each session independent of responding to facilitate contextreward learning, as described previously (Fuchs et al., 2005). Assignment for training in context 1 versus 2 was randomized. Active lever presses resulted in a $2 \mathrm{~s}$ activation of the infusion pump only.

After self-administration training, rats underwent $2 \mathrm{~h}$ extinction sessions on at least 10 consecutive days. During extinction training, responses on either lever had no programmed consequences. Extinction sessions were conducted in context 1 for rats that had been trained to respond for cocaine in context 2 , and in context 2 for rats that had been trained to respond for cocaine in context 1 . To accustom rats to the intracranial infusion procedure, injection cannulas were bilaterally inserted into the guide cannulas for $4 \mathrm{~min}$, but fluid was not infused, before placement into the chamber on extinction day 7. Extinction sessions were terminated when the rats reached the extinction criterion (i.e., $\leq 25$ responses per session on 2 consecutive days).

On the test day, rats received bilateral microinfusions of $\mathrm{B} / \mathrm{M}$ or vehicle into the dlCPu or overlying SS, immediately before placement into the context in which they previously self-administered cocaine. The order of $\mathrm{B} / \mathrm{M}$ and vehicle infusions was counterbalanced across two test sessions based on cocaine intake history. During testing, lever presses were recorded but had no scheduled consequences. Between the two test sessions, the rats underwent additional extinction sessions in the alternate, non-cocaine-paired context until they reached the extinction criterion described above. This resulted in an average $3 \mathrm{~d}$ intertest interval.

Experiment 3: inactivation effects on explicit conditioned stimulusinduced reinstatement of cocaine seeking after extinction. In experiment 2, inactivation of the dlCPu, but not the overlying SS, impaired contextinduced reinstatement of cocaine seeking after extinction training. To examine whether this effect was selective to contextual stimuli and to extend the functional comparison between the $\mathrm{dlCPu}$ and the BLA and dmPFC, experiment 3 assessed the effects of dlCPu inactivation on explicit conditioned stimulus (CS)-induced reinstatement of cocaine seeking after extinction.

New groups of rats $(n=19)$ received guide cannula implants into the $\mathrm{dlCPu}$ or SS and were trained to self-administer cocaine. The house light was illuminated throughout each session. Lever presses on the active lever resulted in a $2 \mathrm{~s}$ activation of the infusion pump and a $5 \mathrm{~s}$ presentation of a CS complex, consisting of activation of the white stimulus light above the active lever and the tone generator $(78 \mathrm{~dB}, 10 \mathrm{kHz})$. On postcocaine days $8-14$, rats underwent $2 \mathrm{~h}$ extinction sessions on a minimum of 7 consecutive days. During extinction training, lever presses had no programmed consequences. On post-cocaine days $1-7$, rats remained in the home cage to maximize the similarity between experiments 1 and 3 in the length of the post-cocaine period that preceded the test of cocaine seeking. To accustom rats to the intracranial infusion procedure, injection cannulas were bilaterally inserted into the guide cannulas for $4 \mathrm{~min}$, but fluid was not infused, before placement into the chamber on extinction day 4. Extinction sessions were terminated when the rats reached the extinction criterion ( $\leq 25$ responses per session during 2 consecutive days). 
Two extinction tests and two reinstatement tests were conducted using a counterbalanced within-subjects design. The extinction tests were conducted to examine the effects of dlCPu or SS inactivation on baseline operant behavior in the absence of the CS, whereas the reinstatement tests were conducted to examine the effects of inactivation on the ability of the lighttone CS complex to reinstate responding. On each test day, rats received bilateral infusion of $\mathrm{B} / \mathrm{M}$ or vehicle into the dlCPu $(n=9)$ or SS $(n=10)$ before placement into the chamber for a $2 \mathrm{~h}$ session. During the extinction test sessions, lever responses had no programmed consequences. During the reinstatement test sessions, active lever responses resulted in $5 \mathrm{~s}$ presentations of the light-tone CS complex in the absence of cocaine reinforcement. Each CS presentation was followed by a $20 \mathrm{~s}$ timeout period. Inactive lever responses had no programmed consequences. The order of $\mathrm{B} / \mathrm{M}$ versus vehicle infusions was counterbalanced across the two test days of each test type based on cocaine intake history. On the days intervening the test days, rats underwent additional extinction sessions until they reached the extinction criterion described above. This resulted in an average $3 \mathrm{~d}$ intertest interval.

Experiment 4: inactivation effects on locomotor activity. Motor-suppressing effects of intracranial treatments can attenuate lever-pressing behavior. To assess possible motor-impairing effects of $\mathrm{B} / \mathrm{M}$ administered into the dlCPu or $\mathrm{SS}$, we examined the effects of $\mathrm{B} / \mathrm{M}$ or vehicle infused into these brain regions on locomotor activity in a novel environment in a subset of the rats from experiments $1-3$. At the minimum $48 \mathrm{~h}$ after the last test of cocaine seeking, locomotor activity was assessed in Plexiglas chambers $(40.8 \times 40.8 \times 37.5 \mathrm{~cm})$ equipped with an array of eight photodetectors and corresponding light sources that emitted photobeams $8 \mathrm{~cm}$ apart $4.5 \mathrm{~cm}$ above the chamber floor. A computerized activity system (San Diego Instruments, San Diego, CA) recorded the number of times neighboring photobeams were broken consecutively by a rat moving in the chamber. Each rat was tested once during a $1 \mathrm{~h}$ session, using a between-subjects design. Before placement into the apparatus, rats received bilateral intracranial infusion of $\mathrm{B} / \mathrm{M}$ or vehicle into the $\mathrm{dlCPu}$ ( $n=8,8$ per group, respectively) or SS ( $n=10,10$ per group, respectively). Assignment to $\mathrm{B} / \mathrm{M}$ versus vehicle pretreatment was randomized.

Histology. Rats were transcardially perfused with PBS and 10\% formaldehyde solution. The brains were dissected out, sectioned in the coronal plane at a thickness of $75 \mu \mathrm{m}$, and stained with cresyl violet (Eastman Kodak, Rochester, NY). The sections were examined under light microscope to determine cannula placement. The most ventral point of each cannula track was mapped onto schematics from the rat brain atlas (Paxinos and Watson, 1997).

Data analysis. ANOVAs were used to analyze lever response. Statistically significant interaction effects were further investigated using Tukey's honestly significant difference tests with the $\alpha$ set at 0.05 .

\section{Results}

dlCPU, but not dmPFC or BLA, inactivation impairs cocaine seeking after abstinence

Cannula placements were verified in the target brain regions (Fig. 1). In experiment 1 , during cocaine self-administration, there
BLA
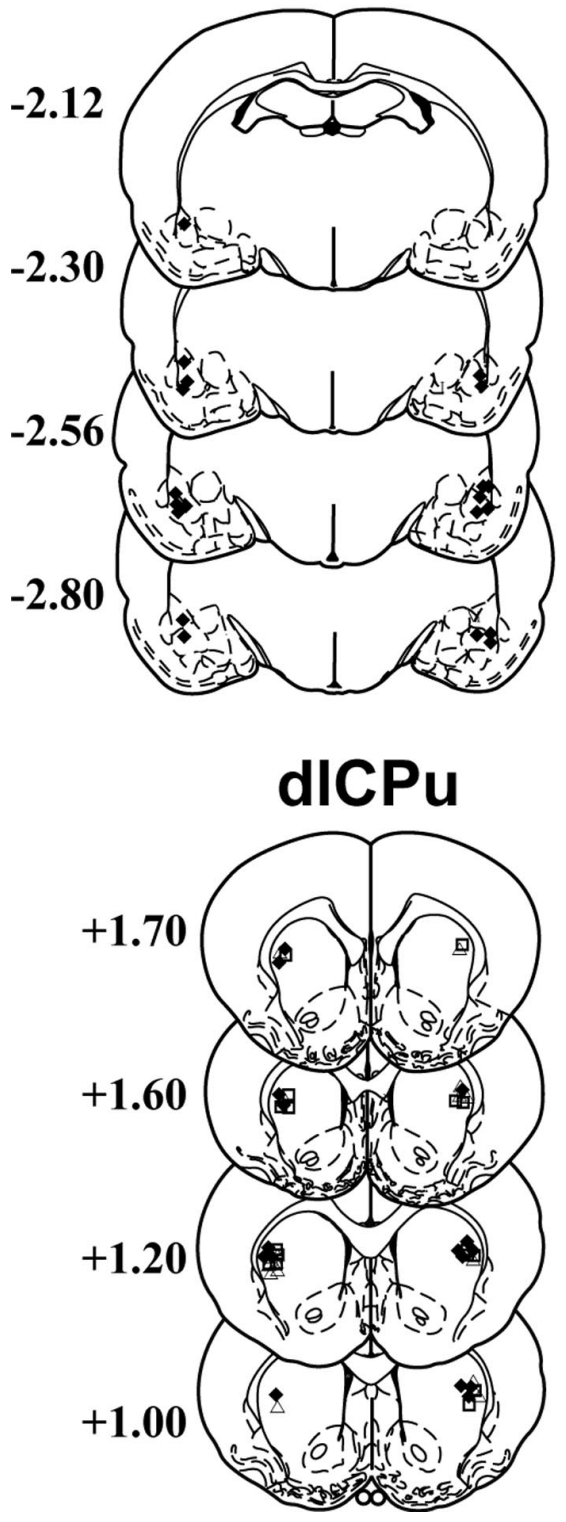

$\mathrm{dICPu}$
dmPFC

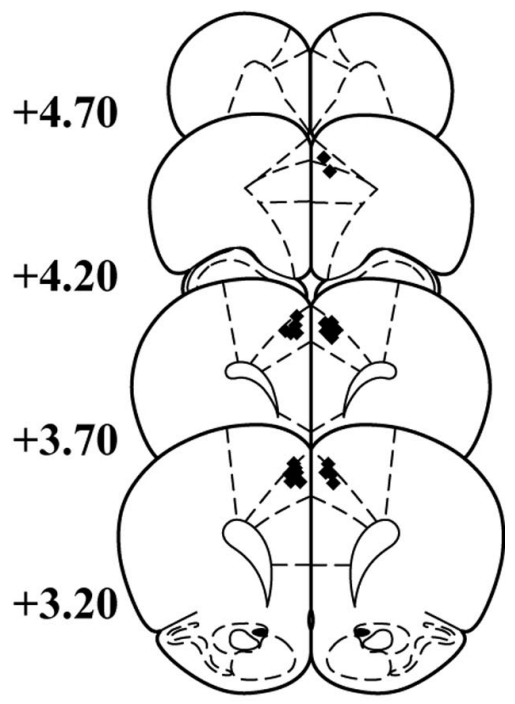

SS

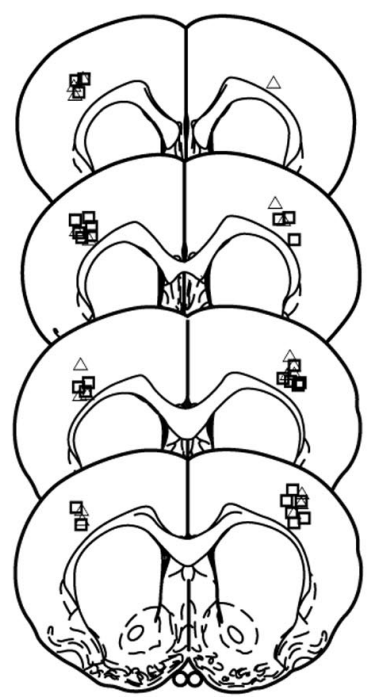

Figure 1. Location of intracranial infusion cannulas, as verified on cresyl violet-stained coronal sections. The symbols represent the most ventral point of the infusion cannula tract for each rat in experiment $1(\diamond)$, experiment $2(\square)$, and experiment $3(\triangle)$. Numbers represent distance from bregma (in millimeters) based on the rat brain atlas of Paxinos and Watson (1997).

was no difference between groups of rats in cocaine-paired active lever responding (ANOVA; group main effect, $F_{(2,26)}=0.79 ; p=$ 0.46 ) or cumulative total cocaine intake (mean \pm SEM, $17.14 \pm$ $0.66 \mathrm{mg} / \mathrm{kg}$ per session; $F_{(2,26)}=0.32 ; p=0.73$ ). Cocaine selfadministration was followed by a $14 \mathrm{~d}$ drug-free abstinence period. Subsequently, reexposure to the drug-paired chamber in the absence of cocaine reinforcement resulted in robust active lever pressing under control conditions (Fig. 2). After vehicle pretreatment administered into the $\mathrm{dlCPu}$, rats exhibited an increase in active lever pressing during reexposure to the cocaine-paired environment relative to self-administration $\left(F_{(2,14)}=6.68 ; p=\right.$ 0.01 ; Tukey's test, $p<0.05)$, and $\mathrm{B} / \mathrm{M}$ inactivation of the $\mathrm{dlCPu}$ attenuated lever pressing relative to vehicle (Tukey's test, $p<$ $0.05)$. Significantly greater responding over self-administration levels was also seen after vehicle pretreatment in the $\operatorname{BLA}\left(F_{(2,18)}=\right.$ 5.80; $p=0.01$; Tukey's test, $p<0.05)$ or $\operatorname{dmPFC}\left(F_{(2,20)}=6.21\right.$; $p=0.01$; Tukey's test, $p<0.05)$. However, $\mathrm{B} / \mathrm{M}$ inactivation of 


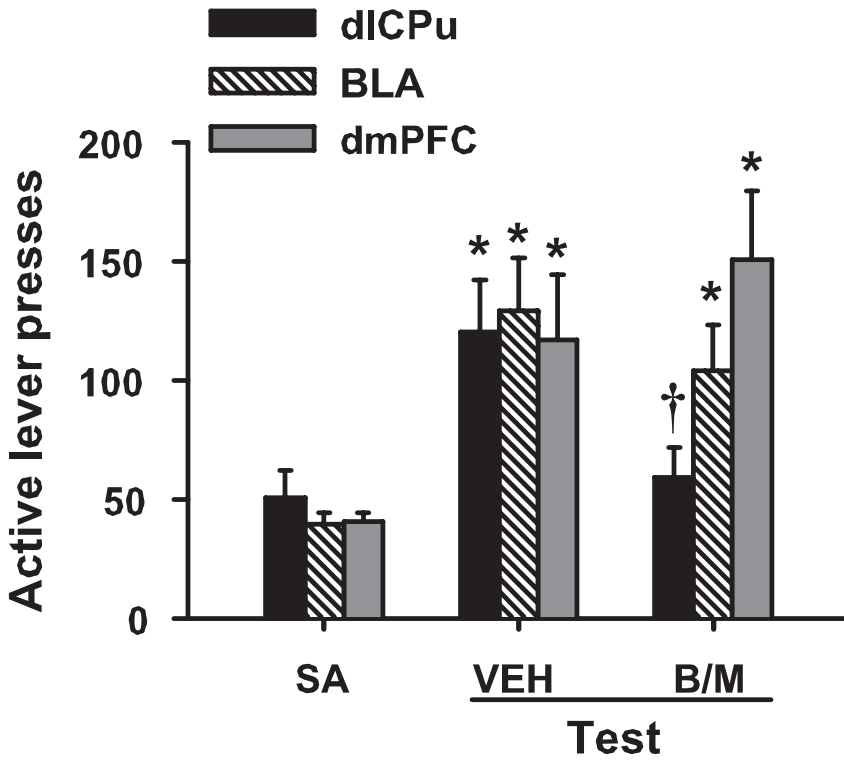

Figure 2. Inactivation of the dICPu attenuates cocaine seeking after abstinence. Active lever presses ( \pm SEM) are shown during self-administration $(S A)$ and the test for cocaine seeking after abstinence. $0 \mathrm{n}$ the test day, rats received pretreatment with intracranial $B / \mathrm{M}$ or vehicle (VEH) and returned to the previously cocaine-paired environment ( $n=8-11$ per region). dICPu-cannulated subjects showed a significant increase in lever pressing relative to selfadministration (Tukey's test, ${ }^{*} p<0.05$ ), and this effect was blocked by inactivation (Tukey's test, $\left.{ }^{\dagger} p<0.05\right)$. In contrast, BLA- and dmPFC-cannulated subjects showed a significant increase in lever pressing relative to self-administration (Tukey's test, ${ }^{*} p<0.05$ ), but this effect was not altered by $B / M$.

these structures failed to alter active lever pressing relative to vehicle. Furthermore, B/M inactivation of any of the three structures failed to alter inactive lever responding relative to vehicle (data not shown).

\section{dlCPU, but not SS, inactivation inhibits context-induced} reinstatement after extinction

In experiment 2 , self-administration was conducted in a distinct context that contained salient, passively presented background stimuli. There was no difference between the groups in total cumulative cocaine intake $\left(t_{(19)}=0.57 ; p=0.55\right)$. After selfadministration in one context, rats underwent extinction in a distinctly different context. They were then reexposed to the cocaine-paired context in the absence of cocaine reinforcement. After vehicle pretreatment administered into the dlCPu or SS, rats exhibited an increase in active lever responding during exposure to the cocaine-paired context relative to extinction (ANOVA; dlCPu, $F_{(2,14)}=10.40, p=0.002$; SS, $F_{(2,24)}=7.27$, $p=0.003$; Tukey's test, $p<0.05$ ) (Fig. $3 a$ ). B/M inactivation of the dlCPu, but not the SS, significantly attenuated active lever responding in the cocaine-associated context relative to vehicle (Tukey's test, $p<0.05$ ). In addition, $\mathrm{B} / \mathrm{M}$ inactivation of either structure failed to alter inactive lever responding relative to vehicle (data not shown).

\section{dlCPU, but not SS, inactivation inhibits explicit CS-induced} reinstatement after extinction

In experiment 3, rats were trained to lever press for cocaine infusions and 5 s presentations of a light-tone CS complex. There was no difference between the groups in total cocaine intake $\left(t_{(17)}=\right.$ 0.35; $p=0.73)$. After self-administration, the rats underwent extinction training in the same context. After extinction, rats were allowed to lever press in the absence of the CS (extinction

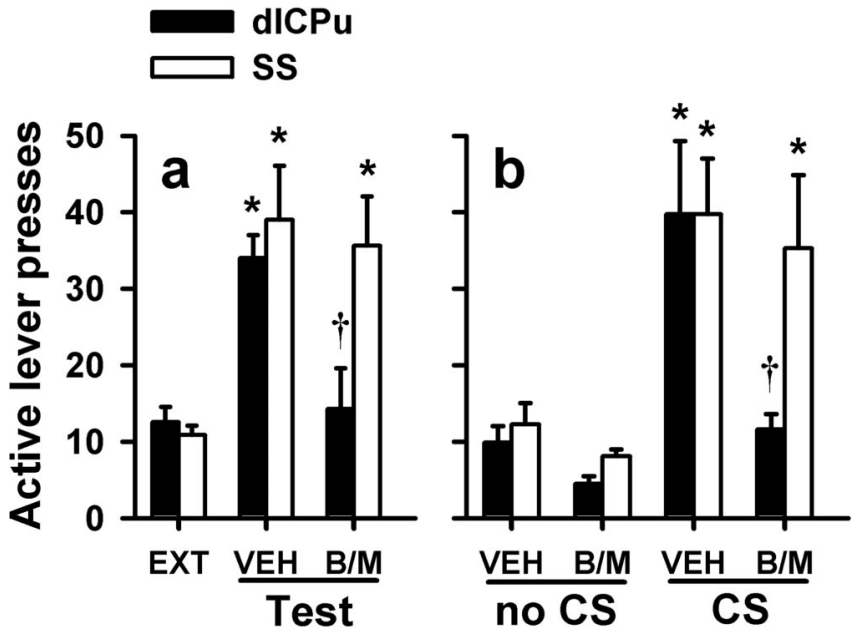

Figure 3. Inactivation of the dICPu attenuates context- or explicit CS-induced reinstatement after extinction. Active lever presses ( \pm SEM) are shown for reinstatement of extinguished cocaine seeking after pretreatment with B/M or vehicle (VEH) into the dICPu or the overlying SS. $\boldsymbol{a}$, Context-induced reinstatement. Rats underwent extinction (EXT) in a context distinctly different from the cocaine-paired context. On the reinstatement tests, rats ( $n=8-13$ per region) were reexposed to the previously cocaine-paired context. Both groups exhibited increased lever pressing relative to extinction after vehicle pretreatment (Tukey's test, ${ }^{*} p<0.05$ ), but only $\mathrm{dICPu}$ inactivation blocked context-induced reinstatement (Tukey's test, ${ }^{\dagger} p<0.05$ relative to vehicle). $\boldsymbol{b}$, (S-induced reinstatement. Rats ( $n=9-10$ per region) underwent extinction in the self-administration chambers in the absence of the $C S$ and were then infused with $B / M$ or vehicle before an extinction test (no $(S)$ or reinstatement test (CS). After vehicle pretreatment, both groups exhibited increased lever pressing in the response-contingent presence, versus the absence, of the CS (Tukey's test, ${ }^{*} p<0.05$ ), but only dICPu inactivation blocked CS-induced reinstatement (Tukey's test, ${ }^{\dagger} p<0.05$ relative to vehicle).

test day) or in the response-contingent presence of the CS (reinstatement test day). After vehicle treatment, all rats exhibited more responses in the presence versus absence of the CS (ANOVA, test day main effect, dlCPu, $F_{(1,7)}=11.46, p=0.02$; SS, $F_{(1,9)}=17.8, p=0.002$ ) (Fig. $3 b$ ). B/M inactivation of the dlCPu attenuated (ANOVA, test day $\times$ treatment interaction, $F_{(1,7)}=$ $5.54, p=0.05$; Tukey's test, $p<0.05)$, whereas inactivation of the SS failed to alter $\left(F_{(1,9)}=0.11, p=0.92\right)$, active lever responding in the presence of the CS relative to vehicle. Additionally, B/M inactivation of either structure failed to alter inactive lever responding relative to vehicle (data not shown).

dlCPU inactivation does not alter locomotor activity

To examine whether the attenuation in active lever pressing after dlCPu inactivation in experiments $1-3$ was attributable to motor suppression, effects of $\mathrm{B} / \mathrm{M}$ versus vehicle pretreatment on locomotion were assessed in a subset of $\mathrm{dlCPu}$ - and SS-cannulated rats that had been used in experiments $1-3$. B/M administered into the dlCPu or SS did not alter locomotor activity relative to vehicle (ANOVA, group effect, dlCPu, $F_{(1,14)}=0.09, p=0.76$; SS, $F_{(1,18)}=1.6, p=0.22$; group $\times$ time interaction, $\mathrm{dlCPu}, F_{(5,70)}=$ $2.23, p=0.06$; SS, $F_{(5,90)}=0.37, p=0.87$; data not shown).

\section{Discussion}

Functional inactivation of the BLA or dmPFC impairs explicit cue-induced and/or context-induced reinstatement of cocaine seeking after extinction (McLaughlin and See, 2003; Fuchs et al., $2004 b, 2005)$. Remarkably, in the present study, inactivation of these structures failed to alter cocaine seeking in a cocaine-paired environment after a $14 \mathrm{~d}$ drug-free abstinence period and no extinction training. In contrast, inactivation of the dlCPU attenuated cocaine-seeking behavior after abstinence and inhibited 
explicit CS- and context-induced reinstatement after extinction training. Impairment in cocaine seeking was not attributable to nonspecific motor effects, because it was stimulus specific (i.e., not present when the CS was absent) and lever specific. Together, these findings suggest that different neural substrates control cocaine seeking after abstinence versus extinction. The dlCPu, a mediator of stimulus-response associations (Jog et al., 1999), exerts more fundamental influence over cocaine seeking after abstinence than brain regions previously implicated in stimulusreward associations. Thus, cocaine seeking after both extinction and abstinence seems to come under the control of stimulusresponse associations.

The finding that the BLA and dmPFC play differential roles in cocaine seeking after abstinence versus extinction likely signifies elemental differences between these behaviors. Cocaine seeking after abstinence may reflect a variety of exogenous variables, including stress, exploratory behavior, and response habit, in addition to cue-induced incentive motivation. Stress is unlikely to have been the critical factor, because rats were handled and exposed to an alternate environment during abstinence to minimize stress-induced cocaine seeking. Furthermore, inactivation of the dmPFC, a structure critical for footshock-induced reinstatement (McFarland et al., 2004), failed to alter responding. It is also unlikely that exploratory behavior explains the results, because $\mathrm{dlCPu}$ inactivation had no effect on locomotion in a novel environment. We suggest that lever pressing after abstinence reflects habitual cocaine seeking, because the dlCPu has been extensively implicated in habit learning (Jog et al., 1999). Consistent with this idea, in primates, cocaine-induced neural activation of the dorsal striatum dramatically increases in the course of chronic cocaine self-administration (Porrino et al., 2004), which may indicate the development of habitual drug use. In cocaine-dependent humans, exposure to cocaine-associated stimuli or cocaine itself triggers neural activation in the striatum (Garavan et al., 2000). Similarly, in rats, response-contingent presentation of a cocaine-paired CS during cue-controlled cocaine seeking results in dopamine release in the $\mathrm{dlCPu}$ (Ito et al., 2002).

The present findings precipitate several interesting questions. First, cocaine seeking was assessed after a $14 \mathrm{~d}$ drug-free period, which is sufficient for the development of "the incubation effect," a time-dependent increase in the magnitude of reward seeking (Tran-Nguyen et al., 1998; Grimm et al., 2001). Habit learning occurs during self-administration training. Therefore, if the dl$\mathrm{CPu}$ contributes to cocaine seeking by mediating response habit, then the effects of dlCPu inactivation on cocaine seeking should be the same before versus after the development of incubation. However, dlCPu inactivation should not alter cocaine seeking before the development of incubation if it acts solely by disrupting incubation. Second, the dlCPu is an element of a larger neurocircuitry yet to be mapped. The substantia nigra is likely included in this circuitry, because dopamine in the dlCPu mediates cuecontrolled cocaine seeking (Ito et al., 2002) and the substantia nigra is the primary source of dopamine in the dlCPu. Future studies will need to investigate these questions.

Cocaine seeking after abstinence may have stronger face validity for cue-induced relapse in humans relative to cocaine seeking after explicit extinction training. However, some nonexplicit extinction learning likely occurs whenever exposure to drugpredictive cues does not coincide with cocaine availability. In preclinical studies, explicit extinction training provides experi- mental control over extraneous variables and isolation of cocaine seeking elicited by the incentive motivational effects of cocaineassociated stimuli, which appear to be overshadowed by other competing behaviors after abstinence. However, extinction training also involves new learning- and experience-based neuroadaptations in several components of the relapse circuitry, including the BLA, nucleus accumbens, and hippocampus (Sutton et al., 2003; Self et al., 2004). Thus, future studies will need to examine whether these learning processes and extinction-induced neuroadaptations may facilitate the ability of these brain regions to regulate cocaine seeking.

\section{References}

Fuchs RA, Evans KA, Parker MC, See RE (2004a) Differential involvement of the core and shell subregions of the nucleus accumbens in conditioned cue-induced reinstatement of cocaine seeking in rats. Psychopharmacology (Berl) 176:459-465.

Fuchs RA, Evans KA, Parker MP, See RE (2004b) Differential involvement of orbitofrontal cortex subregions in conditioned cue-induced and cocaine-primed reinstatement of cocaine seeking in rats. J Neurosci 24:6600-6610.

Fuchs RA, Evans KA, Ledford CC, Parker MP, Case JM, Mehta RH, See RE (2005) The role of the dorsomedial prefrontal cortex, basolateral amygdala, and dorsal hippocampus in contextual reinstatement of cocaine seeking in rats. Neuropsychopharmacology 30:296-309.

Garavan H, Pankiewicz J, Bloom A, Cho JK, Sperry L, Ross TJ, Salmeron BJ, Risinger R, Kelley D, Stein EA (2000) Cue-induced cocaine craving: neuroanatomical specificity for drug users and drug stimuli. Am J Psychiatry 157:1789-1798.

Gerdeman GL, Partridge JG, Lupica CR, Lovinger DM (2003) It could be habit forming: drugs of abuse and striatal synaptic plasticity. Trends Neurosci 26:184-192.

Grimm JW, Hope BT, Wise RA, Shaham Y (2001) Neuroadaptation. Incubation of cocaine craving after withdrawal. Nature 412:141-142.

Ito R, Dalley JW, Robbins TW, Everitt BJ (2002) Dopamine release in the dorsal striatum during cocaine-seeking behavior under the control of a drug-associated cue. J Neurosci 22:6247-6253.

Jog MS, Kubota Y, Connolly CI, Hillegaart V, Graybiel AM (1999) Building neural representations of habits. Science 286:1745-1749.

McFarland K, Kalivas PW (2001) The circuitry mediating cocaine-induced reinstatement of drug-seeking behavior. J Neurosci 21:8655-8663.

McFarland K, Davidge SB, Lapish CC, Kalivas PW (2004) Limbic and motor circuitry underlying footshock-induced reinstatement of cocaine-seeking behavior. J Neurosci 24:1551-1560.

McLaughlin J, See RE (2003) Selective inactivation of the dorsomedial prefrontal cortex and the basolateral amygdala attenuates conditioned-cued reinstatement of extinguished cocaine-seeking behavior in rats. Psychopharmacology (Berl) 168:57-65.

O’Brien CP, Childress AR, Ehrman R, Robbins SJ (1998) Conditioning factors in drug abuse: can they explain compulsion? J Psychopharmacol 12:15-22.

Paxinos G, Watson C (1997) The rat brain in stereotaxic coordinates, Compact Ed 3. New York: Academic.

Porrino LJ, Daunais JB, Smith HR, Nader MA (2004) The expanding effects of cocaine: studies in a nonhuman primate model of cocaine selfadministration. Neurosci Biobehav Rev 27:813-820.

Self DW, Choi KH, Simmons D, Walker JR, Smagula CS (2004) Extinction training regulates neuroadaptive responses to withdrawal from chronic cocaine self-administration. Learn Mem 11:648-657.

Shalev U, Grimm JW, Shaham Y (2002) Neurobiology of relapse to heroin and cocaine seeking: a review. Pharmacol Rev 54:1-42.

Sutton MA, Schmidt EF, Choi KH, Schad CA, Whisler K, Simmons D, Karanian DA, Monteggia LM, Neve RL, Self DW (2003) Extinction-induced upregulation in AMPA receptors reduces cocaine-seeking behaviour. Nature 421:70-75.

Tran-Nguyen LT, Fuchs RA, Coffey GP, Baker DA, O’Dell LE, Neisewander JL (1998) Time-dependent changes in cocaine-seeking behavior and extracellular dopamine levels in the amygdala during cocaine withdrawal. Neuropsychopharmacology 19:48-59. 